\title{
Temperature dependence of charge packet velocity in XLPE cable peelings
}

\author{
L. A. Dissado ${ }^{1}$, S.Zadeh $^{2}$, J.C.Fothergill ${ }^{1}$, A.See $^{3}$ \\ ${ }^{1}$ Department of Engineering, University of Leicester, Leicester (UK) \\ ${ }^{2}$ Work carried out at Department of Engineering, University of Leicester, Leicester (U.K.), now at 19 Gatwick Road, London, \\ U.K. \\ ${ }^{3}$ Now at School of Engineering, University of NewCastle, 2985 Jalan Bukit Merah, Singapore
}

\begin{abstract}
The generation and transit of charge packets in $150 \mu \mathrm{m}$ thick peelings from the insulation of Medium Voltage Cables manufactured using the same XLPE batch have been investigated at a number of different temperatures. Charge packet motion was investigated in peelings taken from cables that have been electro-thermally stressed at $\mathrm{T}=90^{\circ} \mathrm{C}$ for 5000 hours with a Laplacian field $E \approx 20 \mathrm{kV} / \mathrm{mm}$ at the location of the samples. It was found that charge packets were generated by an applied field of $120 \mathrm{kV} / \mathrm{mm}$ when the internal space charge field reached $140-150 \mathrm{kV} / \mathrm{mm}$. Measurements were made in three controlled temperature environments and it was found that the transit time fitted an Arrhenius behaviour with an activation energy of $\sim 1.2 \mathrm{eV}$. This value is similar to estimates made for the deepest traps $(>1.1 \mathrm{eV})$ made from the decay of space charge accumulated at lower fields of $46.67 \mathrm{kVmm}$. It is also close to the activation energy for the conductivity $(\sim 1.25 \mathrm{eV})$ obtained from the dielectric spectroscopy of unpeeled cable sections at $\mathrm{E}_{\mathrm{rms}} \approx$ $0.41 \mathrm{~V} / \mathrm{mm}$, and $\sim 1.15 \mathrm{eV}$ from the dielectric spectroscopy of the peelings $(E=40 \mathrm{~V} / \mathrm{mm})$. It is therefore concluded that the transit of the charge packet is associated with the trap-to-trap transport of charge carriers located in the deepest traps available. The implications of these results for the mechanism of charge packet generation is discussed.
\end{abstract}

\section{INTRODUCTION}

The physical nature of charge packets and their effect (if any) upon the insulation material are vexed questions that have not been resolved since their identification [1]. So far there has been only a few papers [2,3] reporting a systematic investigation of the phenomena, hence although a number of models [1,4-6] have been advanced to describe their formation and transit of the insulation, none have received complete acceptance. Previous work with XLPE cable peelings [2] has shown that a minimum local field, in this case at the anode, was required for the generation of a charge packet. This led to an initiation period during which the internal space charge distribution built up the local field to its required value. The transit of the insulation thickness by the charge packets was slow, and an estimate of the mobility from similar peelings measured at a single temperature [7] gave the activation energy for packet motion in the XLPE peelings as $\sim 0.95 \mathrm{eV}$, and associates the packet with trapped charge. The work reported here carries forward that described in [2] and [7].
Measurements are made at three different temperatures on samples taken from a specific peeling produced by the same programme, so as to determine unambiguously the activation energy for packet transport. This is compared to the value obtained for the dc-conductivity determined by dielectric spectroscopy of both the peelings and a section cut from the same cable from which the peelings were taken. The aim is to build up a body of data on carrier transport and charge packets on a well defined material [8] that can be used to critically assess the various physical mechanisms proposed to explain the packet phenomenon.

\section{MATERIALS}

The insulation material used in this investigation was a specially prepared XLPE batch, which was manufactured into MV-voltage cable with an insulation thickness of $14.6 \mathrm{~mm}$ by two different manufacturers as part of the E.U. ARTEMIS programme [8]. The base polymer was peroxide cross-linked following extrusion. Cable sections and insulation peelings were then used to characterize the insulation [8]. The cables were then electro-thermally stressed for given periods of time, after which sections were cut from them some being investigated as a cable section and some peeled to give insulation peelings for evaluation. All peelings were submitted to a temperature of $50{ }^{\circ} \mathrm{C}$ for 48 hours which reduced the volatile content to negligible levels [8]. A similar conditioning $\left(100{ }^{\circ} \mathrm{C}\right.$ for 72 hours) was applied to the cable sections investigated. All the data reported here was obtained from insulation specimens from a single cable made by one of the two manufacturers that had been stressed at a temperature of $90{ }^{\circ} \mathrm{C}$ for 5000 hours in an ac electric field with a geometrical Laplace value of $20 \mathrm{kV} / \mathrm{mm}$ in the region where the samples were taken.

\section{RESULtS}

Space Charge Measurement Protocol

The space charge dynamics of the peelings was measured using the Pulsed Electro-Acoustic (PEA) technique. The experiments were carried out in a controlled temperature environment, and the experiments at each different temperature were carried out on different samples cut from neighbouring positions on the same peeling tape. 


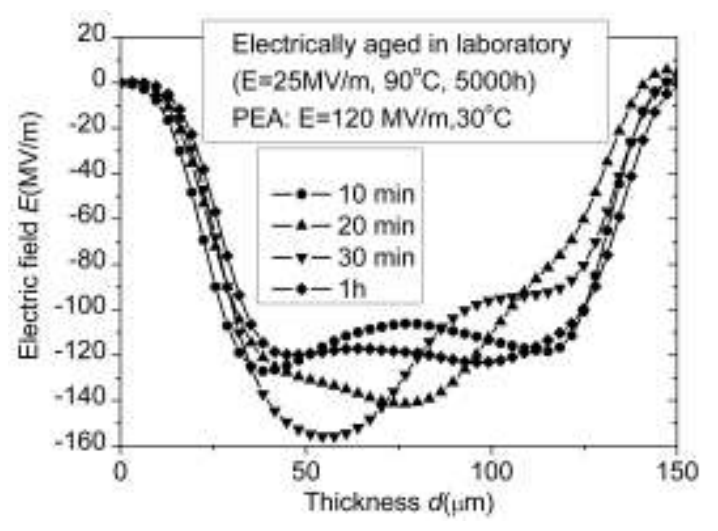

Fig. 1.Electric field distribution at selected times during transit of the sample Charge Packets by a charge packet at $\mathrm{T}=30^{\circ} \mathrm{C}$. Taken from [11]

The results obtained at $\mathrm{T}=20{ }^{\circ} \mathrm{C}$ were similar to those previously reported for peelings from an unaged cable [2]. There is an 'incubation' period of 2.1 hours during which a region of negative space charge extends from the cathode across the sample and enhances the field at the anode. When the anode field reaches a value of $\sim 140 \mathrm{kV} / \mathrm{mm}$ a charge packet is generated that travels across the insulation in about 2.5 hours.

At $\mathrm{T}=30{ }^{\circ} \mathrm{C}$ the incubation time is much shorter $(\sim 5 \mathrm{~min})$ as is the transit time $\left(\mathrm{t}_{\mathrm{t}}\right)$ of the sample $(\sim 40 \mathrm{~min})$. In this case it was difficult to determine clearly an anode field enhancement prior to the initiation of the charge packet, but the field at the front of the packet initially had a value of $\sim 140 \mathrm{kV} / \mathrm{mm}$ (see Fig.1) rising higher as the packet transits to the cathode as in [2].

At $\mathrm{T}=40{ }^{\circ} \mathrm{C}$ it is impossible to distinguish the incubation time $\left(t_{i}\right)$ with our time resolution, (i.e $t_{i} \leq 10 \mathrm{~s}$ ), and the transit of the charge packets is also correspondingly short, with $t_{t} \approx$ 6.25 min., see Fig. 2. Measurements at $\mathrm{T}=50{ }^{\circ} \mathrm{C}$ gave an indication of the transit of a charge packet in the first $30 \mathrm{~s}$ but we were unable to resolve its behaviour.

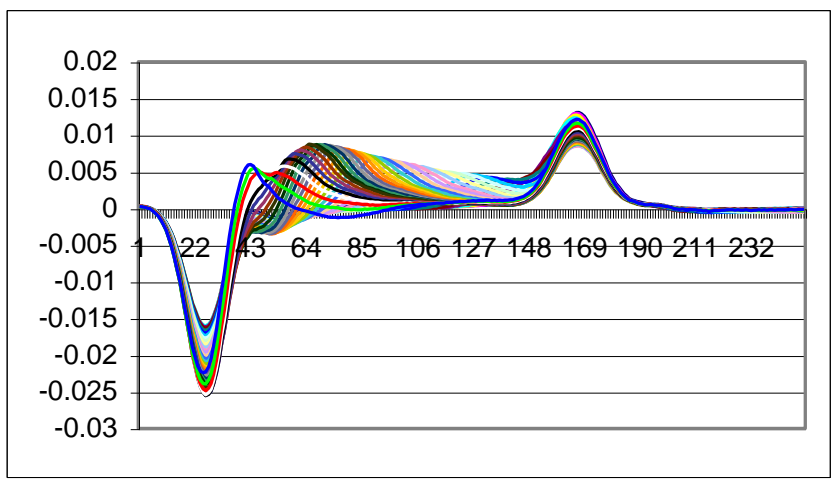

Fig.2. Charge packet transit over first $6.12 \mathrm{~min}$. at $\mathrm{T}=40^{\circ} \mathrm{C}$ (deconvolved but

\section{Packet transit times} uncalibrated data)

A train of charge packets were investigated in [2] and the transit times and time dependence of the electric fields at the

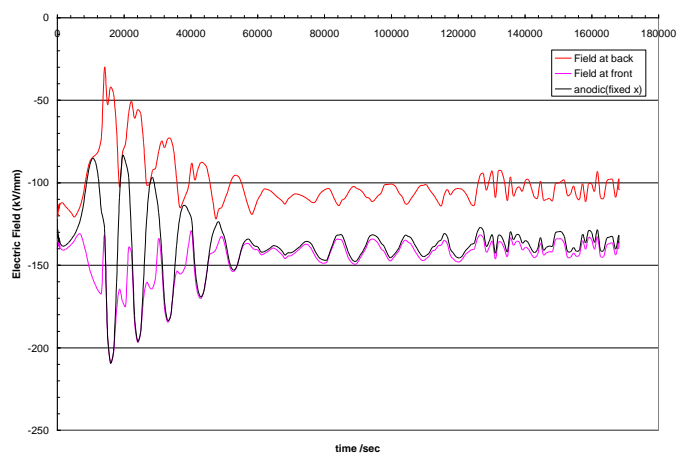

Fig. 3. Anode field and the field at the front and back of the packet. Figure 4 of [2]

front and back of the packet were measured (Fig.3). It was shown that the transit times for different packets in the sequence were the same, as was also found in the current work. This was the case even though the electric field at the front of the packet increased as the packet crossed the sample (as here) and reached different maximum values for different packets in the sequence. Evaluation of the field at the rear of the packet showed that its value reduced as the field at the front increased and that the mean field in the packet was effectively the same for all packets at all times during transit. The value of the mean packet field was shown to be about equal to the minimum field $\left(\mathrm{E}_{\mathrm{p}}\right)$ required for packet initiation for all packets in the sequence, regardless of the magnitude of the packet charge. This suggests that the transit time depends upon the mean packet field and that for a given applied field this is a property of the system. Accordingly an evaluation of the temperature dependence of $t_{r}$ can be expected to yield information about the nature of the packet charge and the way that it moves. An Arrhenius plot of the transit time, Fig. 4, shows that the packet motion is thermally activated with an activation energy of $\sim 1.2 \mathrm{eV}$.

\section{Conductivity Measurements}

Dielectric spectroscopy $\left(10^{-4} \mathrm{~Hz}<\mathrm{f}<10^{5} \mathrm{~Hz}\right)$ was carried out for both peeling samples $(\mathrm{E} \approx 40 \mathrm{~V} / \mathrm{mm})$ and a $300 \mathrm{~mm}$ long cable section $(\langle\mathrm{E}\rangle=0.41 \mathrm{~V} / \mathrm{mm})$, and the contribution of the

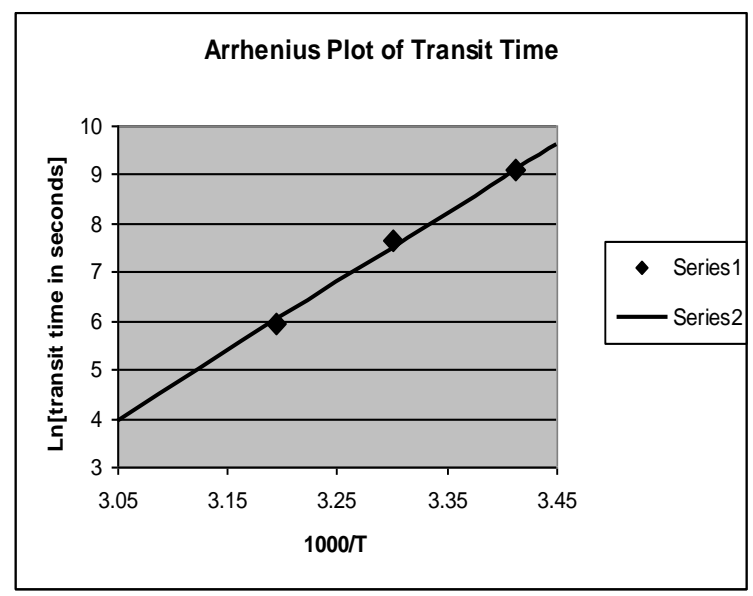

Fig.4. Arrhenius plot of transit time (diamonds) and line fitted to an activation energy of $1.22 \mathrm{eV}$ 


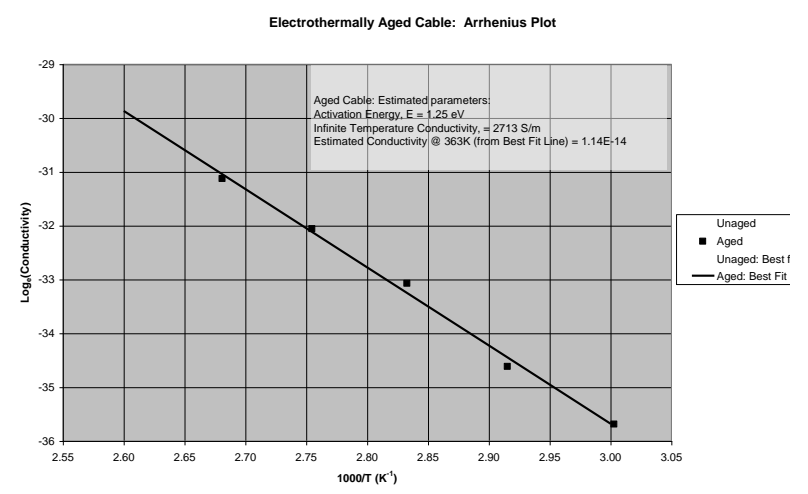

Fig. 5. Arrhenius plot of dc-conductivity in cable section

dc-conductivity identified from the low frequency response where $\chi$ "(f) $\propto 1 / f$. The aim here is obtain information about dc-conductivity at low fields where space charge will not be present to any significant extent, for comparison with the charge transport involved in packets. It was found that the dcconductivity in the peelings had an activation energy of $\sim 1.15$ $\mathrm{eV}$, and in the cable section a value of $1.25 \mathrm{eV}$, see Fig. 5 .

\section{Space Charge Decay}

Measurement of the decay of accumulated space charge following a period of polarization under an applied field can be used to obtain an estimate of the range of trap depths occupied by the space charge $[7,9]$ at fields where charge is accumulated but does not cross the insulation in the form of charge packets. Measurements on samples from the peeling investigated here have already been reported in [10] (denoted as (ETA) electro-thermally aged), where the polarization field was $46.7 \mathrm{kV} / \mathrm{mm}$ and $\mathrm{T} \sim 25^{\circ} \mathrm{C}$. The trap depth range for detrapping times in the available window was estimated to run from $\sim 0.95 \mathrm{eV}$ to a maximum trap depth in excess of $1.1 \mathrm{eV}$.

\section{DISCUSSION}

An explanation of the charge packet phenomena requires the answer to two fundamental questions. One is the organization of injected charge into a packet rather than a homocharge distribution that is maximal at the injecting contact as in the steady-state space-charge-limited (SCLC) situation [12]. The other is the reason why the packet retains its form during the very slow transit of the sample. One point that must be emphasized here is that the conditioning applied to the samples before measurement rules out a role for cross-linking by-products and other volatile chemicals in the packet phenomena observed here, so answers to the questions posed must be sought in terms of the basic polymeric material, its non-volatile additives, and the dielectric/electrode interface.

\section{Packet generation}

Three basic mechanisms have been proposed for the generation of a packet of charge. In [1] it is suggested that the field-dependence of the injection current exhibits hysteresis, whereas in [6] a fluctuation in the injection current produces a packet superimposed upon a SCLC-type homocharge distribution. In contrast [4] relies on acetophenone-assisted field-induced ionization of impurities to generate the packet as an ionization front. The conditioning of our samples precludes such an explanation here. The model of [5] also relies on field ionization, this time of neutral sites via an intermediate state, to give mobile holes and a residual negative layer of ionized sites. The displacement of the layer of ionization provides the moving packet.

In the situation described here the packets appear at the anode when negative heterocharge enhances the field to above a specific value, $\mathrm{E}_{\mathrm{p}}$. Before this value is reached there is little evidence for positive charge injection, and once the packet has been formed there is again little evidence for further positive charge injection until the packet exits at the cathode. This type of packet formation could be regarded as an example of field ionization, which occurs next to the anode because that is where the heterocharge field is maximal. However it is also possible that it is due to a strong enhancement of the injection current that occurs once the anode field exceeds $\mathrm{E}_{\mathrm{p}}$. The injected charge will immediately lower the anode field and reduce the injection current back to a negligible value, leading to an injected charge distribution in the form of a peak next to the anode. In this latter case it would be expected that $E_{p}$ would be dependent upon the type of electrode/polymer interface, whereas if field ionization is the origin of the packet, $\mathrm{E}_{\mathrm{p}}$ should be dependent upon the composition of the insulating material. In either case the amount of charge in the packet will be dependent upon the amount by which $\mathrm{E}_{\mathrm{p}}$ is exceeded at the moment of generation, and thus may vary when a sequence of packet is formed.

\section{Packets during transit}

The retention of packet shape during transit has received two basic explanations. In [5] the packet is a layer of ionization and as such will always retain its shape. In [6] an argument is presented for a negative differential resistance above a given value of field and hence that the speed of charge movement passes through a peak, Fig. 6. The charge speed in the high field at the packet front and in the low field at the packet rear

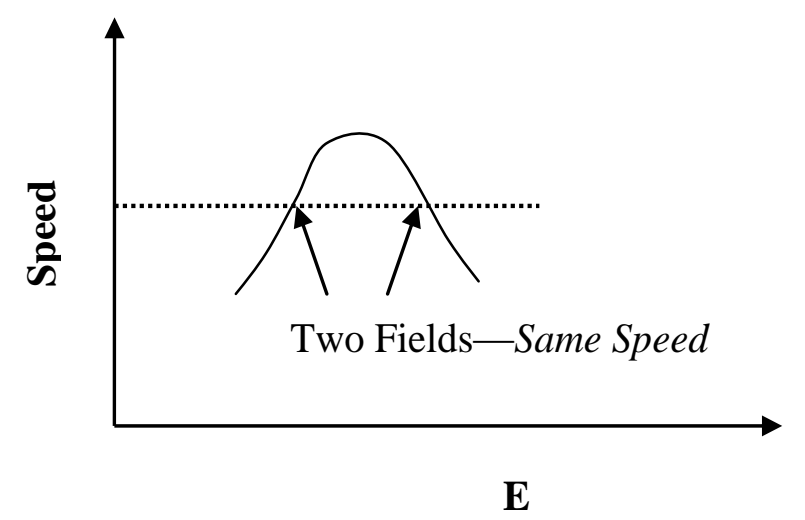

Fig.6. Schematic diagram of charge speed as a function of electric field when a negative differential resistance occurs. 
can thus be made the same. This has the effect that the charge region is kept from spreading as it transits the sample. Such a picture would however mean that the packet should move slower as a consequence of the change in field as the packet transits the sample, see Fig. 3. In addition the packet speeds would be different for the train of packets described in Fig. 3. This explanation of the retention of packet integrity has to be ruled out for the packets considered here as the transit speeds are the same throughout the sequence.

The activation energy obtained for the low-field dcconductivity where samples will not contain any significant amounts of space charge is similar to that of the packet transit time. Since the packet transit speeds are obviously related to bulk material properties rather than those of the electrode interface, it would seem that the activation energy of both packet speed and dc-conductivity must relate to the same kind of property. At low-fields this could either be trap-depths for the carriers or the quantity of intrinsic carriers. The packet is thus associated with the movement of charge between traps or the generation of carriers by ionization. Since field-induced ionization is not likely at the low fields the balance of probability lies with the former possibility. This contention is supported by the space charge decay at high fields below those required to generate package charge. An explanation for the space charge decay in terms of ionized states does not seem possible, and so the range of activation energies deduced $[7,10]$ will relate to the trap depths. A comparison of activation energies for space charge decay and the packet transit time shows that the packet charge must be associated with the deepest filled traps.

At $47 \mathrm{kV} / \mathrm{mm}$ the space charge fills a range of traps up to a minimum trap depth of $\sim 0.93 \mathrm{eV}$ at least, so the question now arises as to why the packet charge is associated with just the deepest traps. One reason may be that there is just a discrete trap level for the polarity of charge injected as a packet. However the very high fields generated and the retention of the packet form suggest an alternative explanation. Once generated the field at the front of the packet is very high. The de-trapping of charge of either polarity will be facilitated, such that the packet charge (here positive) must occupy the deepest traps. Negative charge in the region of the packet will move away from the front of the packet towards to the anode. This will allow the positive packet to gain emphasis as it moves against the background of the negative heterocharge. The lower field at the back of the packet is insufficient to allow further positive charge to move up to the packet, and indeed in our experiments it is insufficient to allow any observable positive charge injection. The packet then is a layer of charges located in the deepest traps, the high field in front of it emptying traps along its path and the low field in its rear preventing further charges joining it, and so the packet does not spread out. Since the transit speed is not determined by the field at the front of the packet but by the field in the middle, where the packet peak is located, it seems that the packet must actually be a thin layer, its broadness being a consequence of the spatial resolution of the technique. The field dependence of the packet advance would then be governed by the field where the plane of the packet is located. It should be noted that the important factor for a field-assisted trap-to-trap hopping is not the field but the potential energy difference of the charge in the traps between which the hopping occurs. In the presence of the field that is acting upon the charge that moves.

\section{CONCLUSIONS}

The transit time of charge packets is a thermally activated process with an activation energy similar in magnitude to that of the low field conductivity and the deepest filled traps at sub-packet fields.

The lack of dependence of the transit time on the front field of the package and the amount of charge in the packet, suggests that the packet is associated with a thin layer of deep trapped charge.

The sample conditioning before measurement indicate that the existence of charge packets is not due to the presence of volatile chemicals.

\section{REFERENCES}

[1] N. Hozumi, H. Suzuki, T. Okamoto, K. Watanabe, and A. Watanabe, "Direct observation of time dependent space charge profiles in XLPE cable under high electric fields", IEEE TDEI, vol.1, pp1068-1076, 1994.

[2] A.See, L.A.Dissado, and J.C.Fothergill, "Electric field criteria for charge packet formation and movement in XLPE", IEEE TDEI, vol 8, pp 859-866, 2001.

[3] M.Fukuma, K. Fukunaga, C. Laurent, "Two-dimensional structure of charge packets in polyethylene under high dc fields", Applied Physics Letters, vol.88, pap 253110, 2006.

[4] N.Hozumi, T. Takada, H.Suzuki, and T. Okamoto, "Space charge behaviour in XLPE cable insulation under $0.2-1.2 \mathrm{MV} / \mathrm{cm}$ dc fields", IEEE TDEI, vol.5, pp82-90, 1998.

[5] K.Kaneko, T.Mizutani, and Y.Suzuoki, "Computer simulation on formation of space charge packets in XLPE films", IEEE TDEI, vol. 6, pp152-158, 1999

[6] J.P.Jones, J.P.Llewellyn, and T.J.Lewis, "The contribution of fieldinduced morphological change to the electrical aging and breakdown of Polyethylene", IEEE TDEI, vol. 12, pp951-966, 2005

[7] G. Mazzanti, G.C.Montanari, and J.M.Alison, "A space charge based method for the estimation of apparent mobility and trap depths as markers for insulation", IEEE TDEI, vol. 10, pp187-197, 2003

[8] J.C.Fothergill, G.C.Montanari, G.C.Stevens, C.Laurent, G.Teyssedre, L.A.Dissado, U.H.Nilsson, G.Platbrood, "Electrical, Microstructural, Physical and Chemical Characterization of HV XLPE Cable Peelings for an Electrical Aging Diagnostic Data Base", IEEE TDEI, vol. 10, pp 514-527, 2003

[9] L.A.Dissado, V.Griseri, W.Peasgood, E.S.Cooper, K.Fukunaga, J.C.Fothergill, "The Decay of Space Charge in a Glassy Epoxy Resin following Voltage Removal”, IEEE Trans. DEI, vol. 13, pp903-916, 2006

[10] A.Tzimas, M.Fu, L.A.Dissado, "Characterization of electro-thermally aged XLPE cable peelings through space charge measurements", Ann.Rep.CEIDP (IEEE Pub. 05CH37702), pp30-33, 2005

[11] M.C.Lanca, M.Fu, E.Neagu, L.A.Dissado, J. Mart Mendes, A.Tzimas, S.Zadeh, "Space charge analysis of electro-thermally aged XLPE cable insulation", J.Non-Cryst.Solids, accepted 2007

[12] A.Many A., and G.Rakavy., "Theory of transient space-chargelimited currents in solids in the presence of trapping" ,Phys.Rev., vol. 126, pp1980-1988, 1962 